\title{
LA CONSTRUCTION D'UNE PRATIQUE POLITIQUE INTERSECTIONNELLE DANS LES LUTTES DES TRAVAILLEUSES DOMESTIQUES AU BRÉSIL
}

\author{
Louisa Acciari, Valeria Ribeiro Corossacz
}

L'Harmattan | « Cahiers du Genre »

2020/2 n 69 | pages 231 à 254

ISSN 1298-6046

ISBN 9782343220413

Article disponible en ligne à l'adresse :

https://www.cairn.info/revue-cahiers-du-genre-2020-2-page-231.htm

Distribution électronique Cairn.info pour L'Harmattan.

(C) L'Harmattan. Tous droits réservés pour tous pays.

La reproduction ou représentation de cet article, notamment par photocopie, n'est autorisée que dans les limites des conditions générales d'utilisation du site ou, le cas échéant, des conditions générales de la licence souscrite par votre établissement. Toute autre reproduction ou représentation, en tout ou partie, sous quelque forme et de quelque manière que ce soit, est interdite sauf accord préalable et écrit de l'éditeur, en dehors des cas prévus par la législation en vigueur en France. Il est précisé que son stockage dans une base de données est également interdit. 


\title{
La construction d'une pratique politique intersectionnelle dans les luttes des travailleuses domestiques au Brésil
}

\section{Louisa Acciari et Valeria Ribeiro Corossacz}

\begin{abstract}
Résumé
Cet article propose une analyse des luttes des travailleuses domestiques au Brésil, à partir de deux recherches de terrain. Nous considérons l'imbrication du racisme, du sexisme et de l'inégalité de classe dans leurs vies et luttes, et dans leur rapport avec les mouvements noir, féministe et les syndicats de travailleurs. Si cette imbrication des rapports sociaux produit des formes d'oppression multiples, elle est également un ressort pour les luttes collectives des travailleuses domestiques leur permettant de former des alliances qui ont mené à la promulgation d'une loi garantissant des droits à la catégorie en 2015. Cependant, le caractère intersectionnel de leurs revendications a également produit des tensions et des conflits avec leurs alliés, limitant la portée de leur succès.
\end{abstract}

INTERSECTIONNALITE - TRAVAILLEUSES DOMESTIQUES BRESIL - SYNDICATS - MOUVEMENTS SOCIAUX 
Le 2 juin 2015, le Congrès brésilien votait la Loi Complémentaire $n^{\circ} 150$ garantissant des droits fondamentaux aux travailleuses domestiques, une catégorie formée principalement de femmes noires, descendantes directes ou indirectes d'esclaves, en situation d'extrême précarité, et jusqu'alors exclue du Code du travail. Cette loi a même été qualifiée de "seconde abolition de l'esclavage» par le gouvernement et certain'e-s député $\cdot \mathrm{e} \cdot \mathrm{s}$. Et pourtant, à peine 3 ans plus tard, le 28 octobre 2018, Jair Bolsonaro, connu pour ses positions misogynes, homophobes, racistes et antidémocratiques, et revendiquant d'avoir été le seul député ayant voté contre la loi des travailleuses domestiques, gagnait les élections présidentielles. Ainsi, alors que l'un des groupes les plus exploités du Brésil obtient une réparation historique de son statut juridique inégal, son plus fervent opposant devient, lui, président de la République. D'une certaine manière, l'élite brésilienne n'a pu accepter la «seconde abolition », voyant dans la tentative d'égaliser les droits des travailleuses domestiques avec les autres secteurs une remise en cause de ses privilèges séculaires.

Cet article $^{1}$ revient sur l'organisation collective des travailleuses domestiques au Brésil, commencée il y a plus de 80 ans; plus précisément, nous analysons leurs luttes en considérant le poids de l'imbrication du racisme, du sexisme et de l'inégalité de classe. La lutte politique des travailleuses domestiques se fonde sur une remise en cause de ces multiples formes d'oppressions, leur permettant de créer des alliances avec d'autres mouvements partageant certaines de leurs identités sociales. Ainsi, nous suggérons qu'une perspective intersectionnelle est nécessaire à la fois pour analyser une position spécifique de marginalité, mais aussi la manière dont cette position est contestée et transformée. Nous explorons plus

\footnotetext{
${ }^{1}$ Bien que cet article soit le résultat d'une élaboration commune, Acciari est l'auteure principale de : «Introduction », « Alliances intersectionnelles avec les mouvements sociaux», et "Limites et conflits dans les alliances intersectionnelles ", et Ribeiro Corossacz, de : "L'intersectionnalité : cadre analytique et outils de mobilisation », « Expériences de l'intersectionnalité au niveau des relations individuelles », et «Conclusion». L'" encadré méthodologique» est commun aux deux auteures.
} 
précisément la trajectoire des syndicats de travailleuses domestiques à travers leurs alliances avec le mouvement noir, le mouvement féministe et les syndicats de travailleurs, ainsi que leurs actions pour transformer les rapports d'oppression de genre, race et classe au niveau des relations individuelles. Nous montrerons que si leur stratégie d'alliances a permis d'aboutir à la promulgation de la loi $\mathrm{n} .150$ de 2015, les rapports de pouvoir à l'œuvre entre les syndicats de travailleuses domestiques et leurs allié-e-s ont amené à un résultat mitigé, voire même décevant, du point de vue des militantes.

\section{Encadré méthodologique}

Cet article s'appuie sur nos expériences de terrain conduites de forme indépendante : Louisa, pendant sa recherche sociologique de doctorat entre 2015 et 2017 et son post-doctorat en cours depuis janvier 2019 (Acciari 2018; 2019), et Valeria pendant une recherche anthropologique menée entre 2013 et 2017 (Ribeiro Corossacz 2017 ; 2019). Louisa s'intéresse plus particulièrement à la formation des syndicats de travailleuses domestiques et à leurs modes d'action, tandis que la recherche de Valeria se focalise sur les harcèlements sexuels de la part des employeurs, les considérant en relation avec les conditions de travail, le racisme et le sexisme. Dans les deux cas, nous sommes entrées en contact avec des syndicats de travailleuses domestiques dans les villes de Recife, Salvador, Rio de Janeiro, Nova Iguaçu, Volta Redonda, São Paulo, Campinas et Franca, affiliés à la Fédération Nationale des Travailleuses Domestiques (FENATRAD), et dans la ville de Natal, où le syndicat n'est pas affilié à la Fédération. Nous avons observé leurs activités au niveau local et national, et conduit des entretiens tant avec des syndicalistes (Louisa 36, Valeria 16) qu'avec des travailleuses non syndiquées (Louisa 45, Valeria 20). $\mathrm{Au}$ Brésil, il est plus courant d'appeler les personnes, même publiques, par leur prénom plutôt que par leur nom complet (par exemple, «Lula»). Aussi, nous n'avons pas toujours eu la confirmation du nom de famille des travailleuses interviewées, et avons décidé de les appeler selon leur préférence exprimée, par leur prénom, à moins qu'elles ne soient des personnalités connues (comme Creuza Maria de Oliveira). 
Il faut également noter que le terme «syndicat» définit ici essentiellement un statut juridique, recouvrant une réalité empirique qui ne s'apparente pas aux autres secteurs : les syndicalistes que nous avons rencontrées sont elles-mêmes travailleuses domestiques, leur travail militant est volontaire, les syndicats ne reçoivent quasiment aucune aide financière et ne comptent que sur un nombre extrêmement faible de membres actives (entre 10 et 30 selon les villes). Il s'agit de structures extrêmement précaires et sous-dotées en ressources humaines. Au niveau national, on estime que seulement $2 \%$ de la catégorie est affiliée à un syndicat (Costa Furno 2016). L'adhésion des travailleuses est volontaire, et le prix reste à la mesure de leurs moyens (équivalent à 1 ou 2 euros). Les adhérentes peuvent participer à toutes les activités, y compris aux élections internes, mais se sont bien souvent toujours les mêmes 10 à 15 personnes que nous voyons aux différents évènements et dans la gestion quotidienne des syndicats. Les syndicats ont par ailleurs l'obligation légale de recevoir les travailleuses de leur catégorie professionnelle en cas de conflits avec l'employeur, que celles-ci soient adhérentes ou pas. On voit donc souvent un nombre assez important de travailleuses passer par les syndicats, sans qu'elles soient pour autant adhérentes ou militantes.

\section{L'intersectionnalité : cadre analytique et outils de mobilisation}

Pour comprendre le travail domestique au Brésil, il faut considérer son lien avec le passé esclavagiste et avec l'origine africaine des esclaves. Après l'abolition légale de l'esclavage en 1888, les esclaves libérées, jusqu'alors confinées à une position de servante dans les familles de colons blancs ou de travailleuses dans les champs des fazendas (grandes propriétés terriennes), ont été insérées dans le marché du travail « libre» dans des positions peu qualifiées (Lauderdale Graham 1989). Pour la majorité, elles ont occupé la fonction de travailleuse domestique et beaucoup de leurs descendantes l'occupent encore de nos jours, dans une condition d'emploi souvent informel et peu ou pas rémunéré. En 2018, plus de cent ans après l'abolition, le Brésil comptait environ 6,2 millions de 
travailleurs domestiques, parmi lesquels $93 \%$ étaient des femmes et $63 \%$ des femmes noires. Cela représente $14,6 \%$ des femmes actives sur le marché du travail, et presque un quart des femmes noires actives, demeurant aujourd'hui encore le premier secteur d'emploi pour ces dernières. Leur salaire moyen est inférieur de $60 \%$ à celui des autres travailleurs, et à peine $29 \%$ d'entre elles possèdent un contrat de travail (IPEA 2019).

Cet « héritage de l'esclavage », combiné à la perception des tâches domestiques comme étant naturellement féminines, a conduit à la non-reconnaissance des travailleuses domestiques comme travailleuses de plein droit (Ávila 2016; BernardinoCosta 2015). En effet, jusqu'à la réforme constitutionnelle de 2013, il s'agissait de la seule catégorie professionnelle exclue du Code du travail, adopté en 1943, et ce, au motif que leur activité serait un service "non économique et sans fin lucrative » (CLT, art. 7). Face à cette situation, les travailleuses domestiques s'organisent depuis les années 30 pour leur reconnaissance comme travailleuses. Cette lutte est encore aujourd'hui vécue par beaucoup d'entre elles comme un combat contre une exploitation liée à l'héritage esclavagiste. Selon une militante du syndicat de Campinas : " la difficulté c'est que les travailleuses domestiques sont un vestige de l'esclavage» (Regina, entretien Valeria, 2015).

La littérature brésilienne sur le travail domestique rémunéré se développe surtout à partir des années 1980, en analysant différents aspects : la relation patronne - employée (Azeredo 1989 ; Kofes 2001 ; Brites 2007), les relations entre féministes et travailleuses domestiques (Castro 1992 ; Melo 1989), le rôle du racisme dans le travail domestique (Gonzalez [1988] 2011; Vieira 1987 ; Goldstein 2003). Ces différentes analyses mettent en évidence les rapports d'oppression et de domination à l'œuvre dans le travail domestique, les reliant aux rapports sociaux de genre, de race et de classe. Les études plus récentes expliquent également l'exploitation et la dévalorisation du travail domestique par l'imbrication de ces différents rapports sociaux, utilisant de manière plus explicite le concept d'intersectionnalité (Hirata 2014, 2016). 
À partir des années 1990 et 2000, on voit se développer une production plus spécifique sur les expériences d'organisation politique des travailleuses domestiques. Quelques études retracent l'histoire de leurs organisations (Chaney et Castro, 1989 ; Bernardino-Costa 2015), tandis que d'autres se focalisent sur l'expérience des militantes les plus connues (Oliveira 2008 ; Pinto 2015). Cependant, ce domaine de la littérature reste relativement moins exploré que les relations de travail, ce qui pourrait être expliqué par une certaine invisibilisation de l'action politique de ce groupe social. Notre recherche contribue donc à développer ce second aspect, se focalisant sur les travailleuses domestiques comme acteur politique. Nous démontrerons que les militantes conçoivent leur oppression comme étant le résultats de rapports sociaux imbriqués de genre, race et de classe, et que cette perspective oriente leur stratégie politique.

Le concept d'intersectionnalité, compris comme l'analyse de l'imbrication de multiples vecteurs d'oppression (Crenshaw 1989, 1991; Hill Collins 2007), nous permet d'analyser à la fois la situation particulière des travailleuses domestiques et leurs réactions face à cette situation d'oppression. Nous comprenons les vecteurs de genre, race et classe comme étant co-constitutifs les uns des autres, et contextualisés. Cela signifie que ces configurations peuvent changer et être transformées par l'action des groupes sociaux. Nous empruntons ici plus spécifiquement la définition proposée par Hill Collins et Bilge (2016), à savoir, l'intersectionnalité comme une pratique politique enracinée dans le vécu des rapports sociaux, visant la transformation sociale, en combattant simultanément le racisme, le sexisme et l'inégalité de classe.

Certaines auteures ont démontré que l'intersectionnalité peut permettre aux groupes marginalisés de rendre compte de leurs formes communes d'oppression (Chun, Lipstiz et Shin 2013 ; Cole 2008), et dans le cas spécifique des travailleuses domestiques, de former des alliances avec d'autres mouvements sociaux (Bernardino-Costa 2015; Nadasen 2015). Nous choisissons donc de nous inscrire dans cette riche littérature qui met en relation mobilisations sociales et intersectionnalité, tout en étant conscientes des débats importants autour du terme et 
dont nous ne pouvons rendre compte en détail dans cet article (voir par exemple Kergoat 2012 ; Lépinard, Fassa et Escoda, 2016). Nous considérons l'intersectionnalité comme l'analyse de l'expérience dynamique de différentes formes d'inégalité, une expérience qui est enracinée dans des contextes spécifiques et marquée par la co-production mutuelle de rapports sociaux. Cette approche permet également de connecter une situation structurelle d'oppression à la production d'identités sociales particulières et situées, qui peuvent être reconnues, contestées ou utilisées par les individus aussi à des fins organisationnelles.

Il faut préciser que si les travailleuses domestiques rencontrées mobilisent les termes de «genre », " race» et « classe», ainsi que les identités sociales «femmes", « Noires » et « pauvres », elles n'emploient pas directement le terme académique d'intersectionnalité. Cependant, nous avons observé que les militantes produisent une analyse de leur oppression en termes de co-production des rapports sociaux, et s'en servent pour construire ce que nous qualifions de pratique politique intersectionnelle. Nous analysons cette pratique à deux niveaux : celui de l'action institutionnelle des syndicats, qui ont construit des alliances avec d'autres mouvements sociaux, ce qui a permis d'aboutir à la promulgation de la loi de 2015 ; et celui individuel, qui vise à transformer la subjectivité des travailleuses domestiques afin de les émanciper des relations d'oppression avec leurs patrons.

\section{Alliances intersectionnelles avec les mouvements sociaux}

L'expérience vécue par les travailleuses domestiques des dynamiques du racisme, du sexisme et de l'exploitation de classe s'est traduite, au long de l'histoire, dans la formation d'alliances stratégiques avec les mouvements sociaux qui partagent certaines de leurs expériences d'oppression: le mouvement noir, syndicaliste et féministe.

\section{Les organisations du mouvement noir}

La première «Association Professionnelle d'Employées Domestiques » a été créée en 1936 par la travailleuse et 
militante Laudelina de Campos Mello (1904-1991), qui faisait partie du Front Noir Brésilien (FNB), fondé en 1931. Les travailleuses domestiques demandaient déjà, à l'époque, l'accès aux droits sociaux et le droit de se syndiquer (Pinto 2015). Durant les années 1940 et 1950, Laudelina de Campos de Mello parvient à organiser, avec l'appui du FNB, des activités culturelles tels que des bals ou déjeuner festifs. Ces activités permettent de réunir les domestiques afin de consolider leur lutte pour l'égalité des droits, en tant que femmes noires et en tant que travailleuses (Pinto 2015). Les liens étroits entre Laudelina de Campos Mello et le mouvement noir donnent encore aujourd'hui un cadre à l'action et au discours des associations de travailleuses domestiques, faisant d'elles un groupe représentatif du racisme structurel au Brésil (Carneiro 2011).

En 1978 est créé le Mouvement Noir Unifié (MNU), dans le but de regrouper toutes les organisations noires existant alors, avec, au début des années 1980, une branche féminine, dont l'une des figures les plus connues est la militante et anthropologue Lélia Gonzalez. Les dirigeantes actuelles du syndicat de Salvador font toujours partie de cette organisation. Le fait que la majorité des travailleuses domestiques soit noire implique que les demandes politiques mises en avant par le mouvement noir affectent directement leurs conditions de vie. Revendiquer l'identité sociale de " femme noire » permet aussi aux dirigeantes d'inscrire la lutte des travailleuses domestiques dans l'histoire de la lutte contre l'esclavage, et de garantir ainsi l'appui du mouvement noir à leur cause. Comme l'affirme Creuza Maria de Oliveira, présidente de la FENATRAD de 2002 à 2016 et actuelle secrétaire générale :

La lutte des domestiques au Brésil, elle a à voir avec le genre, la race et la classe. Il y a également un vestige, ou un processus historique du mouvement contre l'esclavage (entretien Louisa, 2015).

À Campinas, certaines dirigeantes du syndicat font partie du FECONEZU (Festival Comunitário Negro Zumbi), une organisation locale du mouvement noir. Plusieurs militantes ont d'ailleurs été recrutées à travers cette organisation, faisant du syndicat une continuation de leur lutte antiraciste. Anna 
Semião, ancienne présidente du syndicat et première présidente de la FENATRAD de 1997 à 2001, explique :

Je faisais déjà partie du mouvement des femmes noires [quand j'ai été recrutée]. Je savais que c'était la cause de toutes nos difficultés dans la vie. Ce n'était pas parce que nous étions des travailleuses domestiques, mais parce que nous étions noires. Et c'est parce que nous sommes noires, nous sommes des travailleuses domestiques (entretien Louisa, 2016).

Selon elle, c'est l'imbrication des vecteurs d'oppression de genre et de race qui a produit la situation sociale subalterne des travailleuses domestiques. Sans utiliser le terme d'intersectionnalité, Anna Semião fait écho dans son discours aux analyses des chercheuses féministes qui expliquent également le statut particulier du travail domestique par l'intersection des oppressions de genre, race et classe (Gonzalez 1988, Hirata 2014, Castro 1992).

Cependant, peu de travailleuses domestiques, hormis les syndicalistes, sont membres des organisations noires. Il s'agit plutôt d'une alliance entre les deux mouvements autour de questions communes telles que la violence d'Etat, et d'un soutien des principales organisations du mouvement noir envers les demandes spécifiques des travailleuses domestiques, notamment lors des débats autour de la loi de 2015 .

\section{Le mouvement des travailleurs}

Le mouvement des travailleuses domestiques se construit également autour d'une identité et d'un discours de classe. Dans les années 1960, plusieurs associations locales de travailleuses domestiques sont créées avec l'appui des Jeunesses Ouvrières Catholiques (JOC), un courant proche de la Théologie de la Libération au Brésil. ${ }^{2}$ Ces branches locales progressistes de l'église offrent aux travailleuses domestiques une formation politique et un soutien matériel. Cette formation marxiste influencera durablement la production du discours politique des organisations de travailleuses domestiques. Dès le retour de la démocratie en 1988, les associations locales font le choix de se

2 Bulletin d'information: Sindicato dos Trabalhadores Domésticos do Município de São Paulo 
transformer en syndicats et de se regrouper au sein d'une Fédération Nationale (FENATRAD) afin de pouvoir intégrer la Centrale Unique des Travailleurs (CUT), la plus grande confédération nationale au Brésil, directement alliée au Parti des Travailleurs (PT). De cette manière, les syndicats de travailleuses domestiques font le choix de s'insérer dans la lutte de la classe des travailleurs et travailleuses, et insistent sur leur appartenance à cette classe. Un livret écrit en 1989 par le syndicat de Recife et distribué à tous les syndicats locaux affirme :

Les tâches que nous réalisons sont indispensables au reste de la société. Elles garantissent l'hygiène et l'alimentation de milliers de travailleurs; les futurs travailleurs - les enfants. Elles permettent aux hommes et aux femmes, qui travaillent en dehors de leur maison, de récupérer leurs forces de manière quotidienne [...] En effet, notre travail dans les maisons rend possible le travail public du reste de la société. ${ }^{3}$

Les travailleuses domestiques ont ainsi développé un discours positif sur leur travail, comme ayant une valeur sociale tout aussi importante que les autres professions. Cela leur permet de revendiquer des droits en tant que travailleuses et de garantir une place légitime dans le champ de l'organisation syndicale. Toutes les syndicalistes rappellent toujours l'importance du passage de la condition « d'esclave » à celle de «travailleuses ». Il s'agit, d'une part, de redonner une juste valeur à leur travail, le mettant sur un pied d'égalité avec les autres professions, et d'autre part, de garantir l'accès aux droits associés au fait d'être un « travailleur ».

Sa relation organique avec la CUT a fait de la FENATRAD un allié naturel du PT lorsque celui-ci est élu à la tête de l'État en 2002. Les représentantes syndicales gagnent un accès direct au gouvernement, elles reçoivent des financements du PT et de la CUT pour se rendre et séjourner à Brasilia afin de rencontrer ministres et députés, et la FENATRAD siège de manière consultative au Secrétariat de Politiques Publiques pour les

3 Livret sur les droits de la catégorie: Sindomésticos Recife (1989), Domésticas: Uma Categoria da Classe Trabalhadora. Seus Direitos, Suas Lutas, Suas Propostas (p. 7). 
Femmes (SPM) et au Secrétariat pour la Promotion de l'Égalité Raciale (SEPPIR), deux organes de rang ministériel. Les travailleuses domestiques ont ainsi pu être présentes dans un nombre important de consultations, utilisant ces cadres pour mettre en avant leurs revendications et obtenir plus de droits. Leurs demandes font échos à la volonté proclamée du PT de réduire les inégalités sociales et de représenter les plus pauvres. Les travailleuses domestiques deviennent alors un enjeu emblématique des luttes sociales et politiques. Cette stratégie de lobby institutionnel permet l'élaboration d'une réforme constitutionnelle en 2013 (PEC 72), déclarant les travailleuses domestiques égales aux autres catégories de travailleurs, et aboutissant à la promulgation de la Loi Complémentaire $n^{\circ} 150$ en 2015 qui leur garantit un certain nombre de droits du travail, parmi lesquels: l'obligation pour l'employeur de signer un contrat de travail dans les premières 48 heures, le salaire minimum, une limitation de la journée de travail à 8 heures et 44 heures hebdomadaires, une compensation salariale pour le travail de nuit et les heures supplémentaires, l'accès à la retraite et au chômage.

\section{Les mouvements féministes}

Enfin, l'alliance avec les mouvements féministes, dont on pourrait s'attendre à ce qu'elle soit la plus évidente étant donné la surreprésentation des femmes dans le travail domestique rémunéré, s'est pourtant construite avec plus de difficultés que dans le cas du mouvement noir et syndical. Au niveau national, la FENATRAD entretien un partenariat étroit avec le Conseil National des Droits des Femmes (CNDM), l'ONU Femmes, et les militantes du PT. Ces alliances lui ont garanti un appui essentiel lors des débats autour de la loi sur le travail domestique. En effet, la première version de la réforme constitutionnelle de 2013, qui a donné lieu à la loi de 2015, a été formellement proposée par le SPM et a bénéficié du soutien des principales organisations féministes. Travailleuses domestiques et féministes se retrouvent autour d'un projet concret de défense des droits de l'un des principaux secteurs d'emploi féminin. 
Néanmoins, sur le terrain, les relations entre syndicats de travailleuses domestiques et organisations féministes sont marquées par de profondes divisions de race et de classe. En effet, le travail domestique rémunéré permet aux femmes blanches de la classe moyenne de s'émanciper des tâches domestiques et d'éviter les conflits reliés à la répartition des tâches au sein du foyer. Mais, étant un travail déqualifié et non formalisé, il est devenu une forme d'exploitation des femmes pauvres et noires, dans une relation qui est conçue comme étant un problème entre femmes différentes selon leur appartenance de classe et de race (Gonzalez 1984 ; Parreñas 2001; Ehrenreich et Hochschild 2003; Moujoud et Falquet 2013).

Bien que les féministes aient contesté, de façon théorique et politique, cette division socio-sexuelle du travail, les inégalités de race et de classe qui traversent le groupe des « femmes » font que, souvent, les travailleuses domestiques brésiliennes associent le féminisme au milieu social de leurs patronnes: celui de la classe moyenne et blanche. Les dirigeantes du syndicat de Rio de Janeiro observent avec ironie : " qui garde leurs enfants lorsqu'elles manifestent? », tandis que certaines dirigeantes du syndicat de São Paulo affirment que les féministes «s'en fichent des domestiques » (entretiens Louisa, 2016). Les dirigeantes de la FENATRAD ont également affirmé être régulièrement témoins de cas d'employeuses féministes qui ne signent pas de contrat de travail avec leur employée ou «ne paient pas leurs droits » correctement. ${ }^{4}$ Faisant écho aux critiques des féministes noires envers le féminisme blanc (Gonzalez 1988; Bairros 1995; Carneiro 2011), les travailleuses domestiques remettent ici en cause l'universalité de la catégorie «femme», se focalisant sur la relation employeuse/employée. Leurs observations ramènent à la question de comment construire une lutte commune à toutes les femmes dans un contexte d'inégalités sociales parmi les femmes elles-mêmes.

D'autre part, nous avons constaté que la question de la division sexuelle du travail qui assigne aux femmes le travail

\footnotetext{
${ }^{4}$ Voir également les travaux de Castro (1992) qui montrent des observations similaires.
} 
domestique rémunéré et non rémunéré n'est pas envisagée dans la pratique politique et le discours des travailleuses domestiques, alors qu'elle demeure centrale dans la réflexion des chercheuses féministes de classe moyenne et blanches. Cette question est même perçue comme antagonique à leurs intérêts, la revendication d'une meilleure répartition des tâches domestiques entre les sexes étant vécue par certaines travailleuses comme une attaque à leur profession (Sorj 2014). En effet, pour certaines dirigeantes, si les couples de classe moyenne répartissaient de manière égale les tâches ménagères cela pourrait conduire au chômage des travailleuses domestiques. Ainsi, leur lutte ne vise pas à bouleverser le mécanisme qui assigne aux femmes le travail domestique (rémunéré et non rémunéré), mais plutôt à reconnaître le statut de travail aux activités domestiques qu'elles exercent en dehors de leur maison.

\section{Expériences de l'intersectionnalité au niveau des relations individuelles}

Au-delà de l'action institutionnelle des syndicats, nous avons également observé au cours de nos terrains une pratique politique intersectionnelle au niveau des relations individuelles. Le but principal des militantes lorsqu'elles reçoivent les travailleuses dans leur local syndical est que celles-ci prennent conscience de leurs droits en tant que citoyennes et travailleuses, renforçant ainsi ce que nous appelons une «subjectivité politique active». Il s'agit de transformer la relation traditionnelle de subordination imbriquée dans l'héritage colonial et esclavagiste en une relation employeur/employée, dans laquelle la travailleuse a des droits à faire respecter. Ce faisant, les militantes proposent de remettre en cause les «matrices » d'oppression de genre, race et classe qui façonnent la position de subalternité de la travailleuse domestique, et insistent sur le statut social et juridique de la travailleuse comme travailleuse (en opposition à servante ou esclave). 
En effet, les militantes encouragent les travailleuses à ne pas se sentir, et ne pas agir, comme des victimes, mais comme des citoyennes ayant des droits. ${ }^{5}$ Comme le disent plusieurs activistes, il s'agit d'une question d'estime personnelle (autoestima), un terme qui pourrait sembler renvoyer à une dimension purement psychologique, mais qui énonce la conscience que pour ces femmes il est difficile de se voir comme des sujets de droit. Dans les entretiens avec les syndicalistes et militantes, et les interactions observées aux sièges des syndicats entre les activistes et les travailleuses, nous avons pu reconnaître l'effort pour sortir d'une dimension purement psychologique de la dénonciation du manque de respect des patrons, pour se concentrer surtout sur les aspects relatifs aux droits travaillistes. Tout en reconnaissant le côté émotionnel de l'expérience de harcèlement moral et de l'exploitation vécus par ces femmes, les activistes déplacent le plan de l'analyse sur la relation de travail et sur le respect des droits et des comportements professionnels (voir Fish 2017 pour le contexte étasunien).

L'une des techniques employées est d'encourager les travailleuses à amener les patrons au siège du syndicat pour que la relation de travail soit régularisée et les disputes résolues, ou même leur téléphoner pour que l'activiste puisse aborder au téléphone le sujet d'une façon neutre. Ce discours qui cible la dimension professionnelle de la relation entre patrons et travailleuses valorise la position de la femme en tant que travailleuse et citoyenne, et, de cette manière, entre en harmonie avec les politiques d'inclusion sociale des gouvernements du PT qui ont envisagé les groupes les plus défavorisés en leur donnant une perspective d'accès aux droits fondamentaux (éducation, santé, habitation). Dans la pratique concrète des syndicats, ce discours et ces pratiques produisent ainsi l'idée que la citoyenneté, et l'accès aux droits, sont incarnés aussi par des femmes pauvres, peu instruites et non blanches,

\footnotetext{
${ }^{5}$ La Constitution Fédérale de 1988 a mis au centre du nouveau projet social et démocratique l'accès de tous et toutes aux droits sociaux (santé, éducation, travail, sécurité sociale). Cet accès universel aux droits est alors conçu comme une forme d'exercice de la citoyenneté.
} 
bouleversant de la sorte l'imaginaire qui associe la citoyenneté à la blanchité, la classe moyenne et l'éducation de qualité. Dans cette pratique de construction de la subjectivité citoyenne, la dimension de genre entre en compte, mais toujours en lien avec la classe et la race; en effet, l'expérience d'exploitation en tant que femme l'est en tant que femme de classe populaire et noire.

Nous avons également pu observer que l'inégalité de classe est l'élément le plus présent dans les discours des militantes et des travailleuses non syndicalisées. Même s'il s'agit d'un corpus d'entretiens non représentatif d'une catégorie professionnelle très nombreuse, et qu'il n'est pas notre but de généraliser, les femmes que nous avons rencontrées (militantes et travailleuses) ont une vision de leur position sociale et des rapports sociaux quotidiens comme étant marqués avant tout par la classe. La région de résidence (urbaine ou rurale), le quartier et le type d'habitation, la profession, ainsi que la possibilité même d'employer des travailleurs domestiques, sont parmi les indicateurs les plus récurrents utilisés par les travailleuses pour lire la société en termes de classe. La plupart d'entre elles expliquent leur condition sociale et les expériences d'exploitation en se référant aux inégalités de classe, même si plusieurs travailleuses nous ont raconté des relations de travail et personnelles positives avec leurs patrons. Cependant, la dimension de la classe au Brésil est toujours marquée par la race, la classe n'étant pas une catégorie neutre du point de vue de la couleur (Guimarães 2002). Ainsi, lorsque nous abordions la question du racisme, les analyses des travailleuses domestiques se développaient autour de l'imbrication du racisme et inégalité de classe. Claudecéia explique par exemple qu'à l'âge de 22 ans elle avait décidé de reprendre ses études, mais sa patronne lui avait alors dit que si elle voulait étudier, elle devait quitter son travail, et, de plus, se référait à elle en l'appelant de macaca (singe; entretien Valeria, 2015). Son projet personnel d'étudier pour améliorer sa condition sociale se heurte au racisme de sa patronne qui définit la position de son employée comme étant dans le monde animal plutôt que celui de l'humain. Cet exemple nous aide à comprendre comment se réalise, dans les trajectoires individuelles des travailleuses, la co-production des différentes matrices de pouvoir, pour 
reprendre les termes de l'analyse intersectionnelle de Hill Collins (2007).

Les militantes racontent les premières fois qu'elles se sont dirigées au syndicat, quand elles n'étaient encore que des travailleuses non-syndiquées, comme un moment où elles se sont senties accueillies et orientées. Cela est rendu possible par cette compréhension que les militantes ont de l'imbrication des rapports de pouvoir à partir de leur propre vécu, connectant ainsi les expériences individuelles d'oppression et d'exploitation à des rapports de pouvoir structurels. L'accueil (acolher) est un élément très valorisé, et qui se manifeste de différentes manières : donner les renseignements, parce qu'elles savent que « une personne quand elle a l'information ou quand elle commence à avoir l'information elle commence déjà à exiger » (entretien Valeria, 2015), inviter à participer aux activités du syndicat, écouter et soutenir les plaintes, mais aussi rire et employer l'ironie pour nommer les relations de pouvoir. C'est par exemple le cas d'une activiste de Campinas qui faisait des blagues avec les travailleuses en train d'attendre leur tour au siège du syndicat: "si tu veux être domestique, enlève ton fichu. Sans fichu sur la tête, ok? La domestique en fichu c'est fini ! » (entretien Valeria, 2015). À travers la référence au fichu sur la tête, l'activiste rappelle à ces femmes à la fois l'origine coloniale du travail domestique ${ }^{6}$ (et donc nomme la race), et le fait qu'elles ne soient plus des esclaves mais bien des travailleuses.

Cette compréhension de la complexité des conditions matérielles se manifeste aussi dans les cas de harcèlement sexuel perpétrés par les patrons, où les militantes reconnaissent les obstacles que les travailleuses affronteraient lors d'une dénonciation: comme le dit Cleide " c'est la parole des domestiques contre celle des patrons » (entretien Valeria, 2015), à savoir la parole d'une femme pauvre non blanche contre la parole d'un homme blanc de classe moyenne. De plus, ce seront d'autres hommes blancs de classe moyenne qui devront les juger au niveau institutionnel. C'est justement parce

\footnotetext{
${ }^{6} \mathrm{Au}$ Brésil de l'époque coloniale, les esclaves d'origine africaine portaient le fichu ou «turban », « turbante » en portugais.
} 
qu'elles connaissent le poids du racisme, de l'inégalité de classe et de sexe, que les militantes comprennent qu'il puisse être parfois impossible pour les travailleuses harcelées de parler. Bien qu'elles les encouragent toujours à dénoncer, les dirigeantes ne les culpabilisent pas si elles n'y arrivent pas. Dans le cas concret des violences masculines, les travailleuses et syndicalistes reconnaissent également comment la classe et la race produisent des clivages entre femmes. Comme raconté par les travailleuses, l'employeuse préfère souvent défendre son mari plutôt que de donner légitimé au témoignage de son employée. Pour les travailleuses domestiques, il est tout à fait compréhensible que leurs employeuses préfèrent défendre leur mari plutôt que de croire une femme pauvre et noire, démontrant ainsi leur conscience des différences de classe et de race (Ribeiro Corossacz 2019).

Il nous semble donc qu'une dimension importante de la pratique politique intersectionnelle proposée par les syndicats se manifeste dans la capacité des militantes à reconnaître et aborder toutes les expériences d'inégalité présentes dans la vie des travailleuses. Elles s'adressent aux travailleuses domestiques en tant que femmes (harcèlements et violences sexuelles), en tant que noires (expériences du racisme et des discriminations) et en tant que travailleuses pauvres dont les droits ne sont pas respectés par les patrons, et cela, d'une manière qui reconnaît la simultanéité de l'expérience de différentes formes d'oppression.

\section{Limites et conflits dans les alliances intersectionnelles}

Cependant, l'expérience des multiples et simultanées formes d'oppression sociale produit aussi des conflits dans les relations entre les travailleuses domestiques et leurs alliés, tant au niveau politique qu'au niveau professionnel et individuel. Tout d'abord, nous avons constaté une forte désillusion parmi les militantes travailleuses domestiques par rapport à la promulgation de la loi de 2015. Derrière le discours de « seconde abolition de l'esclavage» promu par le gouvernement du PT, cette loi continue de maintenir le statut d'exception des travailleuses domestiques dans le Code du 
travail, ce qui implique qu'un certain nombre de dispositions telles que le droit de grève ou les négociations collectives ne soient pas encore réglementées. De plus, la loi ne leur octroie qu'une liste restrictive de droits du travail (un total de 26 contre 36 pour les autres catégories), perpétuant ainsi le statut juridique inférieur des travailleuses domestiques. Pire encore, la nouvelle législation contient des dispositions permettant aux employeurs de contourner les droits garantis dans la loi, comme par exemple la création du statut de la travailleuse journalière (diarista) qui s'applique aux personnes travaillant moins de 3 jours consécutifs par semaine pour le même employeur. La diarista est considérée comme autonome, et par conséquent, exclue des bénéfices sociaux. Par exemple, en cas de perte d'emploi, la diarista ne peut pas toucher l'assurance chômage car elle n'a pas été formellement licenciée ; il s'agit simplement de la fin de sa prestation de service.

Ceci a provoqué une profonde déception de la part des militantes, comme en témoigne Regina: «ceci n'est pas la loi que nous voulions, elle nous a créé plus de problèmes que de solutions » (entretien Louisa, 2016). Les syndicalistes expliquent le résultat final négatif par leur sous-représentation structurelle dans les institutions. Selon Creuza Maria de Oliveira, «les juges, les législateurs et le gouvernement, les trois pouvoirs, se sont tous des employeurs» (entretien Louisa, 2015). En effet, les députés, composés à $80 \%$ d'hommes blancs pour le mandat de 2014-2018, qui ont amendé et voté la loi $\mathrm{n}^{\circ}$ $150 / 2015$, ont servi avant tout leurs intérêts d'employeurs. Le fait que le taux d'informalité se maintienne si élevé (70\%) 5 ans après la promulgation de la loi, avec une part croissante des diaristas au sein de la catégorie, atteignant près de $45 \%$ en 2018 (IPEA 2019), montre que les droits des travailleuses domestiques demeurent relativement peu effectifs.

Enfin, même si le Congrès est le responsable ultime de la rédaction de la loi, et que la plupart des employeurs font partie de l'élite traditionnellement opposée aux droits des domestiques, les militantes de la FENATRAD s'attendaient cependant à plus de soutien de la part de leurs alliés institutionnels. Or, ils et elles se sont vite alignés au discours de « seconde abolition », contredisant l'analyse des travailleuses 
domestiques, et les laissant isolées dans la lutte pour l'application de la loi. Les dirigeantes nous ont confié de nombreux cas de " camarades » féministes, syndicalistes ou du PT employant une travailleuse domestique sans la déclarer, ou sans lui payer tous les bénéfices auxquels elle a droit. Selon leurs propres termes, comment s'attendre à ce que leurs droits soient respectés et défendus, si même leurs alliés cherchent à éviter les charges sociales? Se joue ici un conflit d'intérêts de classe au sein de la même famille politique : les employeurs, même militants, perçoivent le fait de donner plus de droits aux travailleuses domestiques essentiellement comme une augmentation de leurs dépenses, plutôt que comme une mesure de justice sociale ouvrant l'accès de ses travailleuses à un certain nombre de bénéfices. Les droits des travailleuses domestiques mettent aussi en exergue les rapports d'oppression de genre et de race; ils menacent la division sexuelle et raciale du travail qui permet aux femmes blanches, et à la classe moyenne en général, de s'émanciper des tâches reproductives. Beaucoup de féministes et militants de gauche rencontrés sur le terrain ont opté pour la modalité de la diarista, pourtant fortement critiquée par la FENATRAD, car cela leur permet d'éviter de payer des contributions sociales. Les droits des sujets les plus marginalisés sont ainsi opposés aux droits des employeurs à maintenir un certain niveau de privilèges.

\section{$\therefore$}

Les batailles politiques menées par les travailleuses domestiques brésiliennes ont un caractère radical puisqu'elles touchent à plusieurs systèmes d'inégalité : les rapports sociaux de race, de sexe et de classe. Elles nous invitent à adopter un regard intersectionnel pour voir comment racisme, sexisme et inégalités de classe opèrent simultanément au niveau tant individuel que collectif. À travers un long parcours d'organisation au niveau local, institutionnel et national, les travailleuses domestiques ont obtenu des victoires politiques et législatives importantes reconnaissant leur statut de travailleuses. Cela s'est fait en partie grâce à leur pratique politique de l'intersectionnalité tant au niveau individuel, dans 
les relations émancipatrices créées au sein des syndicats, qu'au niveau des alliances institutionnelles avec d'autres mouvements sociaux.

Cependant, il s'agit de succès inévitablement ambivalents et complexes: les mouvements et les sujets sociaux alliés ne partagent que l'un de leurs vecteurs d'oppression et ne peuvent jamais pleinement représenter les travailleuses domestiques. Les alliés sont aussi des employeurs, blancs et de classe moyenne, ce qui peut limiter la portée des victoires communes. Une fois la loi promulguée, commence alors une bataille plus solitaire pour son application. En effet, respecter les droits des travailleuses domestiques signifie aussi remettre en cause les privilèges de la classe moyenne. Il nous semble ainsi que les résultats des luttes des travailleuses domestiques peuvent produire un projet de justice sociale, et ce, précisément parce que ces luttes touchent à plusieurs formes d'inégalités et d'oppression. Les reconnaître comme travailleuses de plein droit c'est remettre en cause simultanément les divisions de classe, sexuelles et raciales du travail, et repenser la structure hiérarchique de la société brésilienne.

\section{Remerciements}

Les auteures tiennent à remercier les évaluateurs et évaluatrices anonymes pour leurs commentaires sur les différentes versions de ce texte, ainsi que le comité de rédaction des Cahiers du Genre. Un remerciement spécial aux syndicats de travailleuses domestiques au Brésil qui nous ont généreusement accueillies et consacré de leur temps.

\section{Références}

Acciari Louisa (2018). "Paradoxes of Subaltern Politics: Brazilian Domestic Workers' Mobilisations to Become Workers and Decolonise Labour ». Thèse de Doctorat, Londres, London School of Economics and Political Science.

Acciari Louisa (2019). « Decolonising Labour, Reclaiming Subaltern Epistemologies: Brazilian Domestic Workers and the International 
Struggle for Labour Rights ». Contexto Internacional, 41 (1) : 3963.

Ávila Maria Betânia (2016). «O tempo do trabalho doméstico remunerado: entre cidadania e escravidão ». In Abreu Alice Rangel de Paiva, Hirata Helena, Lombardi Maria Rosa (eds). Gênero e trabalho no Brasil e na França: perspectivas internacionais. São Paulo, Boitempo : 137-149.

Azeredo Sandra Maria da Mata (1989). «Relações entre empregadas e patroas: reflexões sobre feminismo em países multiraciais ». In Costa Albertina, Bruschini Cristina (dir.), Rebeldia e submissão. São Paulo, Vértice : 195-220.

Bairros Luiza (1995). «Nossos feminismos revisitados». Estudos Feministas, 2 : 458-463.

Bernardino-Costa Joaze (2015). Saberes Subalternos $e$ Decolonialidade: os sindicatos das trabalhadoras domésticas no Brasil. Brasilia, UnB.

Brites Jurema (2007). « Afeto e desigualdade: gênero, geração e classe entre empregadas domésticas e seus empregadores ». Cadernos Pagu, 29: 91-109.

Carneiro Sueli (2011). Racismo, sexismo e desigualdade no Brasil. São Paulo, Selo Negro.

Castro Mary Garcia (1992). «Alquimia de Categorias Sociais na Produção dos Sujeitos Políticos: gênero, raça e geração entre lideres do sindicato de trabalhadores domésticos em Salvador ». Estudos Feministas, 0 : 57-73.

Chaney Elsa, Castro Mary Garcia (1989). Muchachas no more : household workers in Latin America and the Caribbean. Philadelphia, Temple University Press.

Chun Jennifer, Lipsitz George, Shin Young (2013). « Intersectionality as a Social Movement Strategy: Asian Immigrant Women Advocates ». Signs, 38 (4) : 917-940.

Cole Elizabeth (2008). « Coalitions as a Model for Intersectionality: From Practice to Theory ». Sex Roles, 59 (5/6) : 443-453.

Costa Furno Juliane (2016). A longa abolição no Brasil: transformações recentes no trabalho doméstico. Mémoire de master, Campinas, UNICAMP.

Crenshaw Kimberlé (1989). «Demarginalizing the Intersection of Race and Sex: A Black Feminist Critique of Antidiscrimination Doctrine, Feminist Theory, and Antiracist Politics ». University of Chicago Legal Forum, 1989, 1 : 139-167. 
Crenshaw Kimberlé (1991). « Mapping the Margins: Intersectionality, Identity Politics, and Violence Against Women of Color». Stanford Law Review, 43 (6) : 1241-1299.

Ehrenreich Barbara, Hochschild Arlie (2003). Global woman: Nannies, Maids and Sex Workers in the New Economy. London, Granta Books.

Fish Jennifer (2017). Domestic Workers of the World Unite! A Global Movement for Dignity and Human Rights. New York, NYU Press.

Goldstein Donna (2003). Laughter out of Place. Race, Class, Violence, and Sexuality in a Rio Shantytown. Berkeley, University of California Press.

Guedes Graciele Pereira, Monçores Elisa (2019). «Empregadas domésticas e cuidadoras profissionais: compartilhando as fronteiras da precariedade ». Revista Brasileira De Estudos De População, 36:1-24.

Guimarães Antonio Sérgio Alfredo (2002). Classes, Raças e Democracia. São Paulo, Editora 34.

Gonzalez Leila (2011) [1988]. «Por um feminismo Afro-latinoAmericano ». Caderno de Formação Política do Círculo Palmarino, $1:$ :12-20.

Gonzalez Leila (1984). « Racismo e Sexismo na Cultura Brasileira ». Revista Ciências Sociais Hoje ANPOCS : 223-244.

Hill Collins Patricia (2007). Race, Class and Gender, and Anthology (7th ed.). Belmont, Wardsworth.

Hill Collins Patricia, Bilge Sirma (2016). Intersectionality. Cambridge, Polity Press.

Hirata, Helena (2014). « Gênero, Classe e Raça. Interseccionalidade e Consubstancialidade das Relações Sociais ». Tempo Social, 26 (1) : 61-73

Hirata, Helena (2016). «O cuidado em domicílio na França e no Brasil ». In Abreu Alice, Hirata Helena, Maria Rosa Lombardi (eds.), Gênero e trabalho no Brasil e na França: perspectivas internacionais. São Paulo, Boitempo : 193-203.

IPEA (2019). «Os desafios do passado no trabalho doméstico do século XXI reflexões para o caso brasileiro a partir dos dados da PNAD Contínua ». Textos para Discussão n. 2528. Rio de Janeiro : IPEA.

Kergoat Danièle [2009] (2012). « Dynamiques et consubstantialité des rapports sociaux ». In Se battre disent-elles. Paris, La Dispute : 125-140. 
Kofes Maria Suely (2001). Mulher, Mulheres - Identidade, diferença $e$ desigualdade na relação patroas empregadas. Campinas, Edition Unicamp.

Lauderdale Graham Sandra (1989) «Servants and Masters in Rio de Janeiro: Perceptions of House and Streets in the 1870s ». In Chaney Elsa, Castro Mary Garcia (eds.), Muchachas no More. Household Workers in Latin America and the Carebbean. Philadelphia, Temple University Press : 67-80.

Lépinard Eléonor, Fassa Farinaz, Escoda Marta Roca (2016). L'intersectionnalité : enjeux théoriques et politiques. Paris, La Dispute.

Melo Hildete Pereira de (1989). «Feminists and Domestic Workers in Rio de Janeiro ». In Chaney Elsa, Mary Castro Garcia (eds.), Muchachas no More. Household Workers in Latin America and the Carebbean. Philadelphia, Temple University Press : 245-267.

Moujoud Nasima et Falquet Jules (2013). « Cent ans de sollicitude en France. Domesticité, reproduction sociale, migration et histoire coloniale ». Cahiers genre et développement. Genre, migrations et globalisation de la reproduction sociale, $9: 229-246$.

Nadasen Premilla (2015). Household Workers Unite: the Untold Story of African American Women Who Built a Movement. Boston, Beacon Press.

Oliveira Maria Creuza (2008). «A organização das trabalhadoras domésticas no Brasil ». In Avila M. Betânia, Prado Milena, Sousa Tereza, Soares Vera, Ferreira Verônica (eds.) Reflexões feministas sobre informalidade e trabalho doméstico. Recife, Sos Corpo: 109-116.

Parreñas, Rachel Salazar (2001). Servants of globalization: women, migration and domestic work. Stanford, Stanford University Press.

Pinto Elisabete Aparecida (2015). Etnicidade, Gênero, e Educação: Trajetória de Vida de Laudelina De Campos Mello, Vozes Oprimidas. São Paulo, Anita Garibaldi.

Ribeiro Corossacz Valeria (2017). «Lutte des travailleuses domestiques au Brésil. Racisme, sexisme et inégalités de classe ». Journal des Anthropologues, 150-151 : 159- 180.

Ribeiro Corossacz Valeria (2019). « Sexual harassment and assault in domestic work: An exploration of domestic workers and union organizers in Brazil». The Journal of Latin American and Caribbean Anthropology, 24 (2) : 388-405.

Sorj Bila (2014). « Socialização do cuidado e desigualdades sociais ». Tempo Social, 26 (1) : 123-128. 
Vieira Celma Rosa (1987). «Negra: mulher e doméstica considerações sobre as relações sociais no emprego doméstico ». Estudos Afro-Asiáticos, 14 : 141-158. 